\title{
Control of Molar Mass Distribution by Polymerization in the Analytical Ultracentrifuge
}

\author{
Andreas Spinnrock and Helmut Cölfen*
}

\begin{abstract}
Molar mass distributions are of high interest in macromolecular chemistry because they directly determine the physical and chemical properties of polymers. A principal approach to obtain and control the shape of broad molar mass distributions is adjusting the initiator concentration in free radical polymerizations. A controlled gradient of the initiator concentration should potentially lead to tailored molar mass distributions. Here we use analytical ultracentrifugation (AUC) to adjust and measure a macroinitiator's concentration gradient. Subsequent photopolymerization of a uniformly distributed monomer leads to desired chain length distributions. Resulting distributions are described and calculated by a Schulz-Flory approach. The desired concentration profiles are simulated in advance and can be detected anytime by the optical systems in the centrifuge. Therefore, tailored broad molar mass distributions can now be produced using predictions from simulations using the established theory of AUC.
\end{abstract}

Since the beginning of polymer chemistry, control of chain length distributions and molar mass distributions has been a highly investigated and discussed topic. ${ }^{[1,2]}$ The properties of polymers directly depend not only on the average molar mass but also on the width and shape of the molar mass distribution. ${ }^{[3,4]}$ Tensile and impact strength are specifically determined by short molecules, solution viscosity and low shear melt flow are mainly governed by the middle-sized chains, and melt elasticity is predominately influenced by the longest chains. ${ }^{[3]}$ Therefore, control of molar mass distributions allows control of polymer function such that tailored polymer materials can be produced with tailored physicochemical properties to exactly meet the materials demands. In addition, polymers with a tailored molar mass distribution are promising in areas like nanolithography, filtration, and thermoplastic elastomers. ${ }^{[5,6]}$

In free radical polymerizations, the obtainable molar mass distributions are limited due to termination reactions like recombination and disproportionation. Therefore, as the most narrow limit, only dispersities between 1.5 (only recombination) and 2.0 (only disproportionation) can be achieved.$^{[1]}$ In the last 25 years some powerful techniques have been developed to obtain narrow dispersities in radical polymer-

[*] A. Spinnrock, Prof. Dr. H. Cölfen

Physical Chemistry, University of Konstanz

Universitätsstrasse 10, Box 714, 78457 Konstanz (Germany)

E-mail: helmut.coelfen@uni-konstanz.de

Homepage: https://www.chemie.uni-konstanz.de/coelfen/ izations (dispersity $<1.5$ ). The three most promising controlled radical polymerization techniques are atom-transfer radical polymerization (ATRP), ${ }^{[7,8]}$ reversible addition-fragmentation chain-transfer polymerization (RAFT) ${ }^{[9,10]}$ and nitroxide-mediated polymerization (NMP). ${ }^{[11,12]}$ For these reactions, controlled and narrow Poisson distributions are obtained for the molar mass distributions. ${ }^{[13]}$ A few methods have been developed to control molar mass distributions with higher dispersities. One strategy is to blend polymers with different molecular weights to get multimodal distributions. ${ }^{[14,15]}$ These systems lack a continuous distribution and their preparation is tedious because several polymer samples must be synthesized. Recently, Fors et al. reported a modular strategy to control narrow polymer molar mass distributions in nitroxide-mediated polymerization reactions by changing the addition rate of the initiator during the reaction. ${ }^{[5]}$

In contrast, in this this work we control molar mass distributions with high dispersities by free radical polymerization. Broad molar mass distributions are of great technical importance and have tremendous potential to tailor different mechanical properties, for example, a polymer could already contain inherent plasticizers. Different from the common use of analytical ultracentrifugation (AUC) to determine hydrodynamic and thermodynamic properties of macromolecules $^{[16,17]}$ and nanoparticles, ${ }^{[18]}$ ultracentrifugal fields were applied here in a new method to control broad molar mass distributions directly upon synthesis in the analytical ultracentrifuge. With the well-established thermodynamic theory of AUC, ${ }^{[19]}$ a concentration gradient of any dissolved compound in a centrifugal field can be simulated in sedimentation equilibrium (relevant for this work) as well as sedimentation velocity experiments. ${ }^{[20,21]}$ This can be exploited to simulate the concentration distribution of a macromolecular initiator in a nonsedimenting monomer. Therefore, by tuning the macromolecular initiator concentration gradient, various molar mass distributions should become accessible. This makes it possible to obtain broad molar mass distributions, which can be precisely simulated and experimentally tailored by AUC. In the end, the desired broad molar mass distributions can be designed with the computer in advance.

The investigated system consists of the macromolecular photoinitiator Genopol BP-2, the hydrogen-donor methyl diethanol amine, the monomer methyl acrylate, and the solvent THF. The centrifugal force in an ultracentrifugal field leads to the sedimentation of the macromolecular photoinitiator within the nonsedimenting monomer (Figures S1 and $\mathrm{S} 2$ in the Supporting Information). ${ }^{[22]}$ When the desired concentration gradient is adjusted, the rotation process is stopped and a photopolymerization process is started by a $15 \mathrm{~W}$ Xe flash lamp (Figure 1). Depending on the ratio of 


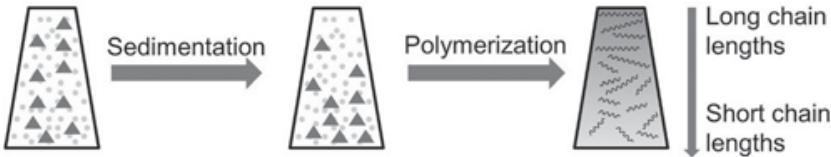

Figure 1. Schematic view of the polymerization process in the AUC cell. The macromolecular photoinitiator (triangles) sediments and forms a concentration gradient in the nonsedimenting monomer (light gray dots). Subsequent photopolymerization leads to different chain lengths depending on the ratio of initiator to monomer at each position.

the monomer concentration to the initiator concentration, polymers with different chain lengths are formed at each radial position. A higher concentration of the initiator and thus a lower ratio of monomer to initiator leads to shorter chains and vice versa. ${ }^{[1]}$ Thus, the macroinitiator concentration gradient leads to a broadening of the molar mass distribution compared to those in reference experiments without application of the ultracentrifugal field (Figure 2). The dispersity was increased from 1.8 to 2.2 when a rotational speed of $60000 \mathrm{rpm}$ was applied.

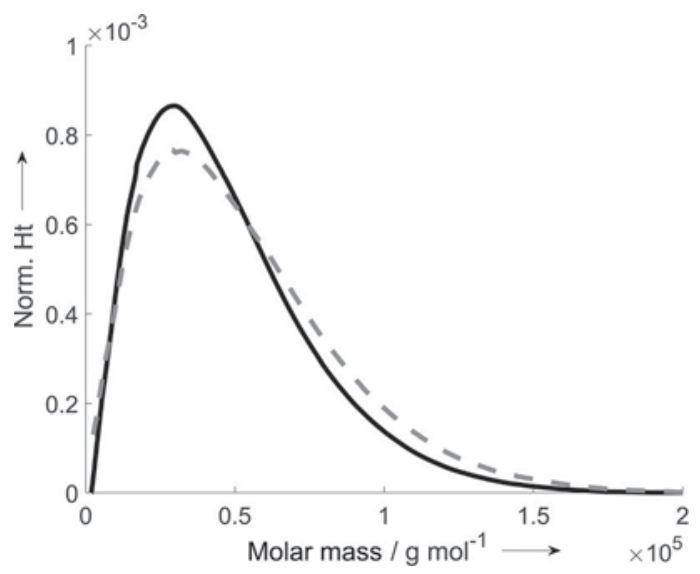

Figure 2. Molar mass distribution of poly (methyl acrylate) after $20 \mathrm{~min}$ polymerization determined by gel permeation chromatography after universal calibration. Distribution without ultracentrifugal field (solid black line) and with ultracentrifugal field at $60000 \mathrm{rpm}$ (dashed gray line).

Mathematically, the kinetic length $v$ describes the chain length of polymer molecules produced by free radical polymerizations [Eq. (1)]. ${ }^{[1]}$

$v=\frac{k_{p}}{\sqrt{f k_{t} k_{d}}} \frac{[M]}{\sqrt{[I]}}$

Here $k_{p}$ is the polymerization rate constant, $f$ is the efficiency factor, $k_{t}$ is the termination rate constant, $k_{d}$ is the initiation rate constant, $[M]$ is the monomer concentration, and $[I]$ is the initiator concentration. Reference experiments for different photoinitiator concentrations were performed to determine the kinetic prefactors for the investigated reaction system. They follow the inverse square-root dependency of the chain length on the initiator concentration (Figure S3). For high initiator concentrations, the observed molecular weights are higher than expected. The reason for this deviation can be that the initiator is not $100 \%$ active.

To generate an initiator concentration gradient in the ultracentrifuge, sedimentation equilibrium experiments were performed. The concentration gradient of the macroinitiator was detected by interference optics and simulated with the software Ultrascan II. ${ }^{[21]}$ The simulated and the detected concentration gradients are in good agreement (Figures S4S6). The initiator concentration shows an exponential gradient, as expected for sedimentation equilibrium runs. ${ }^{[22]} \mathrm{High}$ rotational speed leads to steeper concentration profiles due to higher centrifugal force. For a starting initiator concentration of $6 \mathrm{mg} \mathrm{mL}^{-1}$, the concentration is in the range from 1.9 to $13.6 \mathrm{mg} \mathrm{mL}^{-1}$ at $50000 \mathrm{rpm}$ (Figure S4) compared to a range from 1.1 to $19.7 \mathrm{mg} \mathrm{mL}^{-1}$ at $60000 \mathrm{rpm}$ (Figure S5).

To predict the resulting chain length distribution, the kinetic chain length was calculated with the prefactors from Figure S3 for each radial position of the concentration profiles. Distributions after polymerization were calculated by a Schulz-Flory approach ${ }^{[2]}$ with a mixture of disproportionation and recombination. The molar mass distributions for concentrations $<5 \mathrm{mg} \mathrm{mL}^{-1}$ were described by a SchulzFlory fit for recombination because these fits had a high coefficient of determination in reference experiments with initiator concentrations $<5 \mathrm{mg} \mathrm{mL}^{-1}$ (Figure S7). In terms of mass, the Schulz-Flory distribution for the termination of chains by recombination is described by Equation (2).

$\frac{m_{p}}{m}=0.5 P^{2} a^{P}(1-a)^{3}$

For concentrations $>5 \mathrm{mg} \mathrm{mL}^{-1}$ a Schulz-Flory fit for termination by disproportionation was used, because these fits had a high coefficient of determination in reference experiments with initiator concentrations $>5 \mathrm{mg} \mathrm{mL}^{-1}$ (Figure $\mathrm{S} 8$ ) [Eq. (3)].

$\frac{m_{p}}{m}=P a^{P}(1-a)^{2}$

Here $P$ is the degree of polymerization and $a$ is the probability of the growth reaction.

These models had high coefficients of determination $(>99 \%)$ in reference experiments with different initiator concentrations without ultracentrifugal fields (Figures S7 and S8 and Table S1). At each value of the concentration profile from Figures S4-S6, a Schulz-Flory chain length distribution was plotted from the kinetic chain length and all distributions were added up to obtain the overall chain length and thus the molar mass distribution. The simulated chain length distributions show good correlations with the distributions determined by gel permeation chromatography after universal calibration (Figure 3). An increase in the rotational speed from $50000 \mathrm{rpm}$ (Figure $3 \mathrm{~A}$ ) to $60000 \mathrm{rpm}$ (Figure 3 B) leads to an increase in the dispersity from 1.9 to 2.2 (Figure 3D). A higher initiator concentration ( 2.3 to $38.9 \mathrm{mg} \mathrm{mL}^{-1}$, Figure S6) leads to shorter chain lengths, as expected due to the kinetic chain length (Figure 3C,D). Thus, the chain length distribu- 


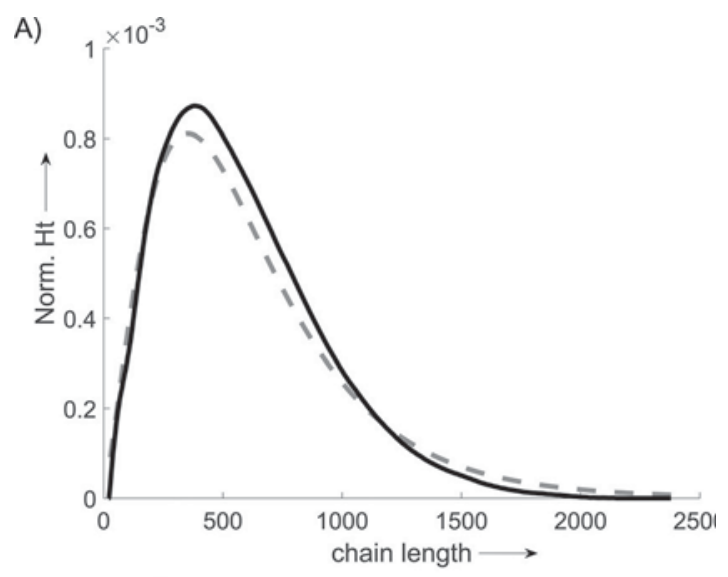

B) $8 \times 10^{-4}$

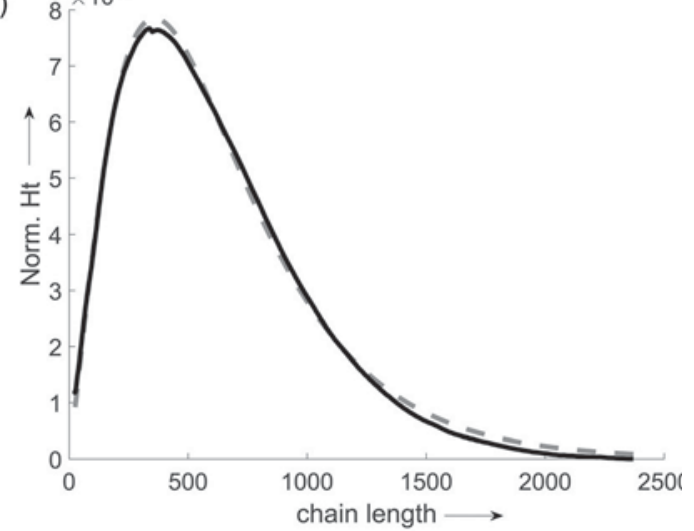

C) $8^{\times 10^{-4}}$
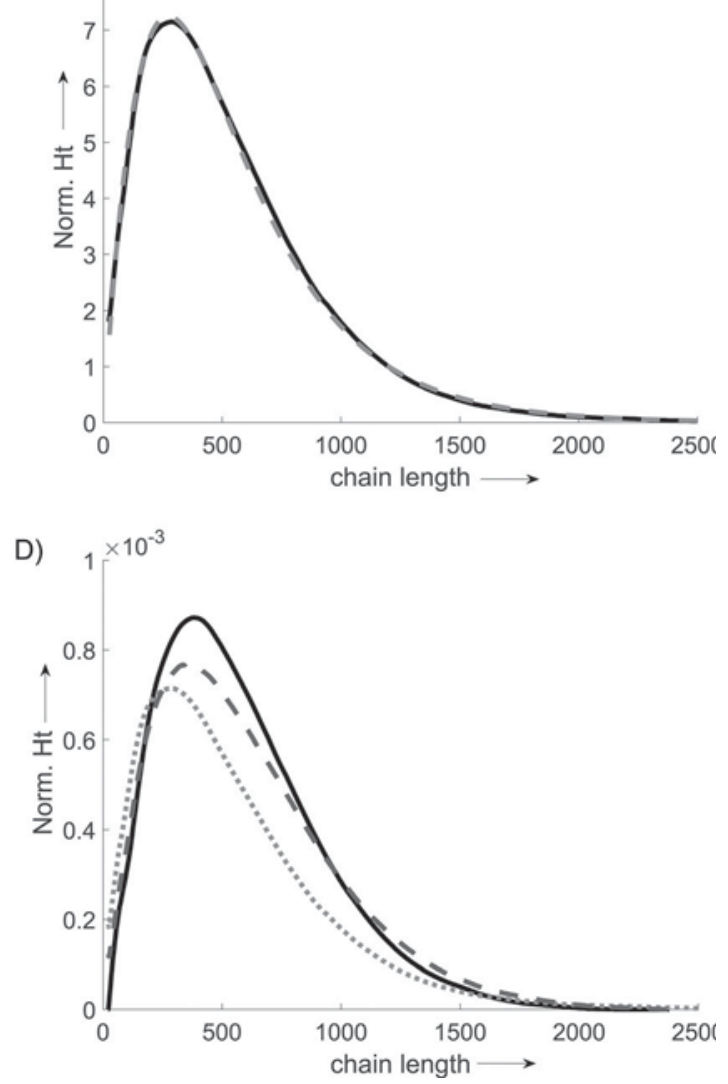

tion can be predicted in advance. Simulation allows the production of targeted distributions.

With the limitations of a maximum rotational speed of $60000 \mathrm{rpm}$ and a limited sedimentation coefficient due to the size of the employed photoinitiator Genopol BP-2, a maximum dispersity of 2.3 was achieved. Steeper initiator concentration gradients would lead to broader chain length distributions. For that, initiators with higher molar masses are needed. Macroinitiators with molecular weights between 1500 and $4000 \mathrm{~g} \mathrm{~mol}^{-1}$ would lead to steeper concentration gradients, for example, from 0.02 to $78.2 \mathrm{mg} \mathrm{mL}^{-1}$ for $2000 \mathrm{~g} \mathrm{~mol}^{-1}$ (Figure S9). Therefore, broader chain length distributions (e.g dispersity of 3.7 for an initiator with a molecular weight of $4000 \mathrm{~g} \mathrm{~mol}^{-1}$ ) and different shapes can be produced (Figure 4). Depending on the molecular weight of the initiator and the rotational speed, the width of the molar mass distributions and the length of the high molar mass tail can be controlled.

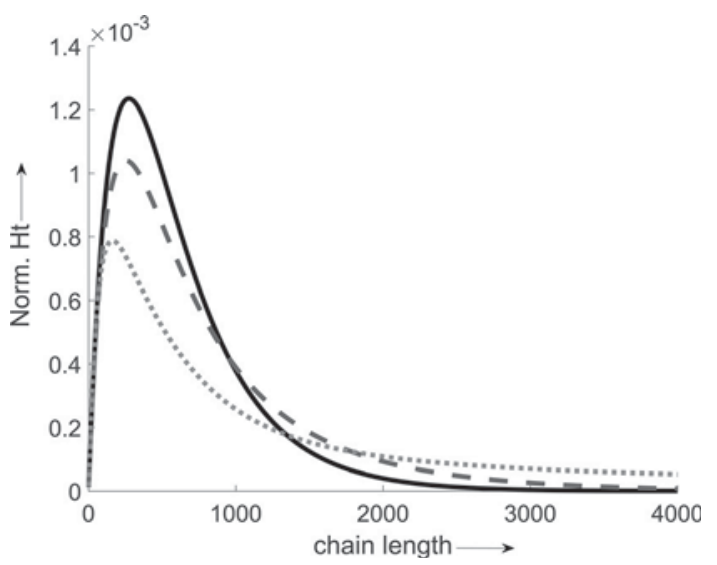

Figure 4. Simulated chain length distributions for polymerization with macroinitiators with different molecular weights. $M=1500 \mathrm{~g} \mathrm{~mol}^{-1}$ (solid black line), $M=2000 \mathrm{~g} \mathrm{~mol}^{-1}$ (dashed dark gray line), $M=4000 \mathrm{~g} \mathrm{~mol}^{-1}$ (dotted light gray line).

The advantage of using an analytical ultracentrifuge to produce the desired distribution is that the concentration gradient is detected by the optical system and that different concentration profiles are possible depending on the performed experiment. For a more general application of the presented work, the initiator concentration does not need to be adjusted by ultracentrifugal fields, but can also be controlled by diffusion or any other fluid mixing processes with known thermodynamics or kinetics suitable to make realistic predictions. The diffusion coefficient is determined by established methods like field flow fractionation ${ }^{[23]}$ and

Figure 3. A)-C) Resulting chain length distributions calculated from GPC measurements (solid black line) and simulated with the SchulzFlory approach (dashed gray line); A) at $50000 \mathrm{rpm}$ and an initiator concentration of $6 \mathrm{mg} \mathrm{mL}^{-1}, \mathrm{~B}$ ) at $60000 \mathrm{rpm}$ and an initiator concentration of $6 \mathrm{mg} \mathrm{mL}^{-1}, \mathrm{C}$ ) at $60000 \mathrm{rpm}$ and an initiator concentration of $12.5 \mathrm{mg} \mathrm{mL}^{-1}$. D) Comparison of chain length distributions calculated from GPC measurements for (A) (solid black line), (B) (dashed dark gray line), and (C) (dotted light gray line). 
analytical ultracentrifugation. ${ }^{[22]}$ A solution with a photoinitiator or thermal initiator can be superimposed on a solution with the monomer. The diffusion process can be described by Fick's laws of diffusion ${ }^{[24]}$ and by that calculated at any time. The polymerization is started at the desired point in time either by illumination or temperature. Therefore, different molar mass distributions can be obtained with less instrumentation and effort.

In conclusion, a new method has been developed to control molar mass distributions by adjusting the macroinitiator concentration in ultracentrifugal fields and subsequent photopolymerization. The resulting molar mass distributions were simulated by a Schulz-Flory approach. By applying different centrifugation velocities, a variety of desired molar mass distributions can be produced. By transferring the process to concentration control through diffusion or any other type of controlled mixing, which can be done in any desired reaction vessel, the concept can potentially be used in any chemical laboratory.

\section{Experimental Section}

For details, see the Supporting Information. Briefly, for the photopolymerization methyl diethanol amine, methyl acrylate, THF, and Genopol BP-2 were mixed and filled in an AUC Ti double-sector cell. The desired concentration gradient was adjusted by sedimentation in the AUC and polymerization for 20 minutes was started with a Xe flash lamp.

\section{Acknowledgements}

The authors acknowledge funding by the Carl Zeiss Foundation for A.S. We thank L. Bolk for GPC measurements.

\section{Conflict of interest}

The authors declare no conflict of interest.

Keywords: analytical ultracentrifugation .

molar mass distribution - photochemistry · polymerization . radical reactions

[1] K. Matyjaszewski, T. P. Davis, Handbook of radical polymerization, Wiley-Interscience, Hoboken, 2002.
[2] P. J. Flory, Principles of polymer chemistry, Cornell University Press, Ithaca, 1953.

[3] D. W. Van Krevelen, K. Te Nijenhuis in Properties of Polymers, 4th ed., Elsevier, Amsterdam, 2009, pp. 7-47.

[4] N. A. Lynd, M. A. Hillmyer, M. W. Matsen, Macromolecules 2008, $41,4531-4533$.

[5] D. T. Gentekos, L. N. Dupuis, B. P. Fors, J. Am. Chem. Soc. 2016, $138,1848-1851$.

[6] K. Matyjaszewski, Y. Gnanou, L. Leibler, in Macromolecular Engineering, Wiley-VCH, Weinheim, 2007, pp. 1-6.

[7] K. Matyjaszewski, J. Xia, Chem. Rev. 2001, 101, 2921-2990.

[8] K. Matyjaszewski, Macromolecules 2012, 45, 4015-4039.

[9] J. Chiefari, Y. K. Chong, F. Ercole, J. Krstina, J. Jeffery, T. P. T. Le, R. T. A. Mayadunne, G. F. Meijs, C. L. Moad, G. Moad, E. Rizzardo, S. H. Thang, Macromolecules 1998, 31, 5559-5562.

[10] G. Moad, J. Chiefari, Y. K. Chong, J. Krstina, R. T. A. Mayadunne, A. Postma, E. Rizzardo, S. H. Thang, Polym. Int. 2000, 49, $993-1001$.

[11] L. Tebben, A. Studer, Angew. Chem. Int. Ed. 2011, 50, 50345068; Angew. Chem. 2011, 123, 5138-5174.

[12] J. Nicolas, Y. Guillaneuf, C. Lefay, D. Bertin, D. Gigmes, B. Charleux, Prog. Polym. Sci. 2013, 38, 63-235.

[13] T. E. Patten, J. Xia, T. Abernathy, K. Matyjaszewski, Science 1996, 272, 866-868.

[14] A. Noro, M. Iinuma, J. Suzuki, A. Takano, Y. Matsushita, Macromolecules 2004, 37, 3804-3808.

[15] G. Hadziioannou, A. Skoulios, Macromolecules 1982, 15, 267 271.

[16] T. M. Laue in Current Protocols in Protein Science, WileyInterscience, New York, 1996.

[17] P. Schuck, Basic principles of analytical ultracentrifugation, CRC, Taylor \& Francis Group, Boca Raton, 2016.

[18] K. L. Planken, H. Cölfen, Nanoscale 2010, 2, 1849-1869.

[19] H. Fujita, Foundations of Ultracentrifugal Analysis, Wiley, New York, 1975.

[20] P. Schuck, Biophys. J. 2000, 78, 1606-1619.

[21] B. Demeler, Anal. Ultracentrifugation Techniques Methods 2005, 210-230; B. Demeler in Analytical Ultracentrifugation: Techniques and Methods, Royal Society of Chemistry, London, 2005, pp. 210-230.

[22] W. Mächtle, L. Börger, Analytical ultracentrifugation of polymers and nanoparticles, Springer, Berlin, 2006.

[23] H. Cölfen, M. Antonietti in New Developments in Polymer Analytics I (Ed.: M. Schmidt), Springer, Berlin, Heidelberg, 2000, pp. $67-187$.

[24] A. Fick, Ann. Phys. 1855, 170, 59-86. 